\title{
Potassium-magnesium Imbalance Causes Detrimental Effects on Growth, Starch Allocation and Rubisco Activity in Sugarcane Plants
}

\section{Ariani Garcia}

Sao Paulo State University

Carlos Alexandre Costa Crusciol ( $\nabla$ carlos.crusciol@unesp.br)

Sao Paulo State University

Ciro Antonio Rosolem

Sao Paulo State University

João William Bossolani

Sao Paulo State University

Carlos Antonio Costa Nascimento

Sao Paulo State University

James Mabry McCray

University of Florida

André Rodrigues dos Reis

Sao Paulo State University

Ismail Cakmak

Sabancı University

Keywords:

Posted Date: March 1st, 2021

DOl: https://doi.org/10.21203/rs.3.rs-244222/v1

License: (9) (1) This work is licensed under a Creative Commons Attribution 4.0 International License. Read Full License

Version of Record: A version of this preprint was published at Plant and Soil on January 16th, 2022. See the published version at https://doi.org/10.1007/s11104-021-05222-2. 


\section{Abstract}

The authors have requested that this preprint be removed from Research Square. 